\title{
Meandered UC-EBG structure for a reduction of the mutual coupling in a patch antenna array
}

\author{
Son Trinh-Van and Keum Cheol Hwang ${ }^{\text {a) }}$ \\ Division of Electronics and Electrical Engineering, Dongguk University-Seoul, \\ Seoul, 100-715, Korea \\ a)kchwang@dongguk.edu
}

\begin{abstract}
A meandered uniplanar compact electromagnetic bandgap structure is proposed. Meandered microstrip lines between adjacent cells are used to increase the equivalent inductance. As a result, the dimension of the proposed EBG structure is more compact and its frequency band-gap is wider than that of the conventional uniplanar EBG structure. Two patch arrays with and without an EBG structure were designed, fabricated, and tested to validate the performance of the proposed EBG structure. The measured results demonstrated that a mutual coupling reduction of $4 \mathrm{~dB}$ is achievable in a patch array with the proposed EBG structure.
\end{abstract}

Keywords: electromagnetic band-gap, meander-line, mutual coupling, antenna arrays

Classification: Microwave and millimeter wave devices, circuits, and systems

\section{References}

[1] D.-B. Hou, S. Xiao, B.-Z. Wang, L. Jiang, J. Wang, and W. Hong, "Elimination of scan blindness with compact defected ground structures in microstrip phased array," IET Microw. Antennas Propag., vol. 3, no. 2, pp. 269-275, March 2009.

[2] M. Salehi, A. Motevasselian, A. Tavakoli, and T. Heidari, "Mutual coupling reduction of microstrip antennas using defected ground structure," 10th IEEE Singapore Int. Conf. Communication Systems, pp. 1-5, Oct. 2006.

[3] Q.-R. Zheng, Y.-Q. Fu, and N.-C. Yuan, "A novel compact spiral electromagnetic band-gap (EBG) structure," IEEE Trans. Antennas Propag., vol. 56, no. 6, pp. 1656-1660, June 2008.

[4] Z. Iluz, R. Shavit, and R. Bauer, "Microstrip antenna phased array with electromagnetic bandgap substrate," IEEE Trans. Antennas Propag., vol. 52, no. 6, pp. 1446-1453, June 2004.

[5] E. Rajo-Iglesias, O. Quevedo-Teruel, and L. Inclan-Sanchez, "Mutual coupling reduction in patch antenna arrays by using a planar EBG structure and multilayer dielectric substrate," IEEE Trans. Antennas Propag., vol. 56, no. 6, pp. 1648-1655, June 2008. 
[6] H. S. Farahani, M. Veysi, M. Kamyab, and A. Tadjalli, "Mutual coupling reduction in patch antenna arrays using a UC-EBG superstrate," IEEE Antennas Wireless Propag. Lett., vol. 9, pp. 57-59, Feb. 2010.

[7] A. Farahbakhsh and G. Moradi, "Design a low mutual coupling microstrip array antenna with non regular polygonal patches," IEICE Electron. Express, vol. 7, no. 17, pp. 1271-1275, Sept. 2010.

[8] T. Liu, X. Y. Cao, J. J. Ma, and X. Wen, "Enhanced bandwidth uniplanar compact electromagnetic bandgap structure with coplanar meander line inductance," Electron. Lett., vol. 44, no. 4, pp. 260-261, Feb. 2008.

[9] F. Yang and Y. Rahmat-Samii, Electromagnetic Band Gap Structures in Antenna Engineering, p. 59, Cambridge Univ. Press, 2009.

[10] D. Sievenpiper, L. Zhang, R. F. J. Broas, N. G. Alexopolous, and E. Yablonovitch, "High-impedance electromagnetic surface with a forbidden frequency band," IEEE Trans. Microw. Theory Tech., vol. 47, no. 11, pp. 2059-2074, Nov. 1999.

[11] S. D. Assimonis, T. V. Yioultsis, and C. S. Antonopoulos, "Computational investigation and design of planar EBG structures for coupling reduction in antenna applications," IEEE Trans. Magn., vol. 48, no. 2, pp. 771-774, Feb. 2012.

\section{Introduction}

Recently, the design of a miniaturized array antenna for unmanned aerial vehicles (UAVs) has attracted attention in communication and military applications. Placing elements of the antenna array close to each other or using a thick substrate with high permittivity are the conventional schemes for reducing the total dimensions of the array. However, these methods significantly increase the surface wave excitation. In the microstrip antenna array, the surface wave also increases the mutual coupling between elements, causing undesirable effects such as scan blindness [1]. Therefore, reducing the mutual coupling has become an important issue in the design of a microstrip antenna array. Several configurations have been proposed to suppress the mutual coupling. In $[1,2]$, defected ground structures (DGSs) that served as a band-rejected filter were applied to reduce the mutual coupling. Nevertheless, these DGSs also acted as radiating slots that increased the back lobe level.

Various EBG configurations have also been proposed to reduce the mutual coupling because the primary attribute of the EBG structure is its bandgap characteristic, which suppresses the propagation of the surface wave. A spiral-like EBG structure with vias had been used for this purpose [3]. Other uniplanar EBG (U-EBG) structures without vertical vias have also attracted much attention due to their easy fabrication $[4,5]$. A multi-layer dielectric superstrate incorporated with U-EBG structures has been employed to reduce the overall dimension of the antenna array [6]. In another study [7], GA-enhanced non-regular polygonal patches were used to achieve low mutual coupling. In spite of the fact that EBG cells when placed on the superstrate layer reduce the element separation and the mutual coupling of patch antenna 


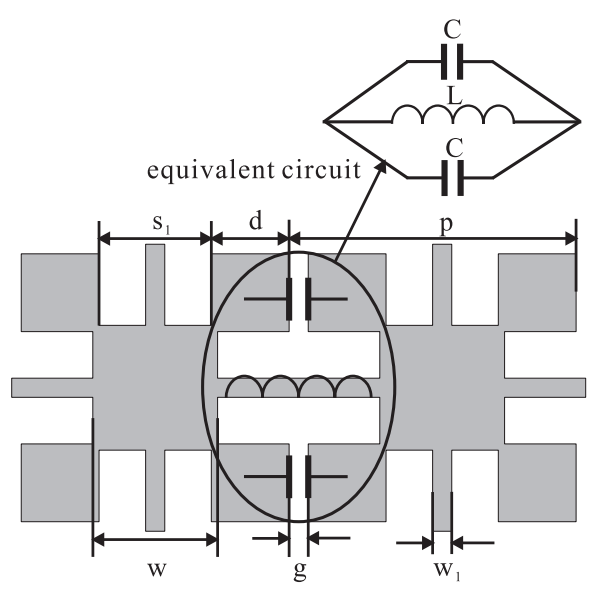

(a)

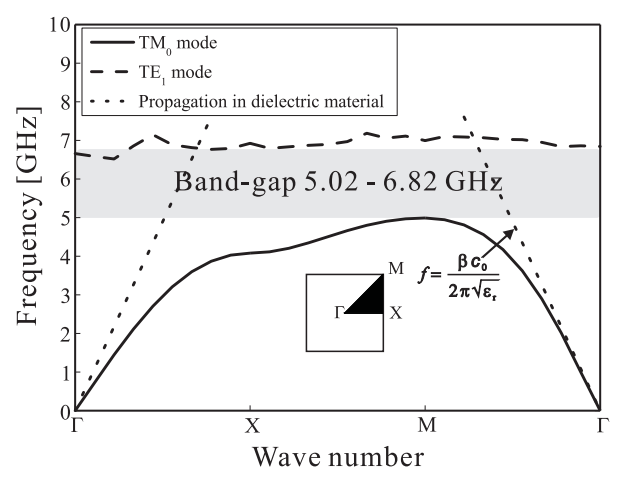

(c)

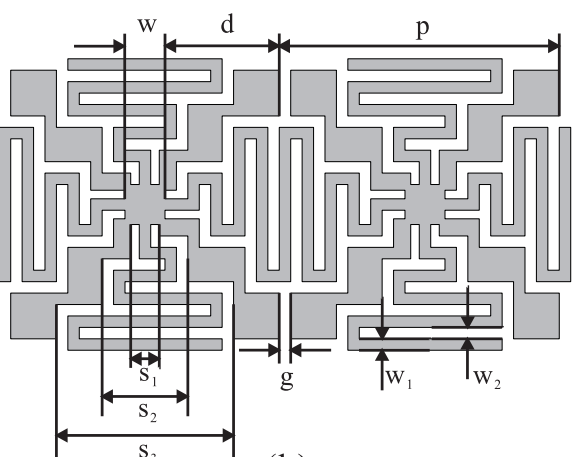

(b)

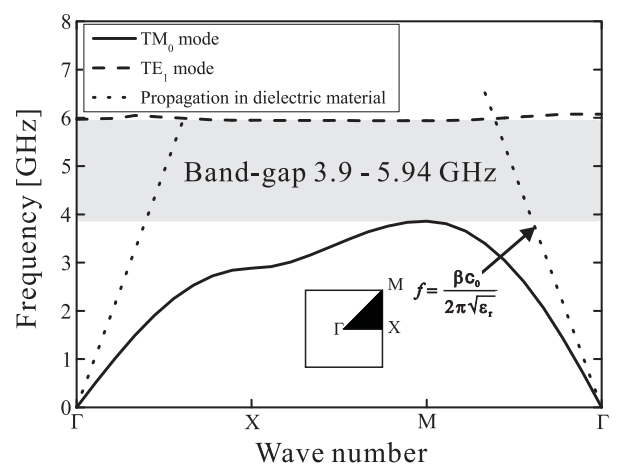

(d)

Fig. 1. Geometry of the EBG structures and dispersion diagrams: (a) conventional U-EBG structure and the equivalent circuit (b) meandered UC-EBG structure (c) $k-\beta$ dispersion diagram for the conventional U-EBG structure (d) $k-\beta$ dispersion diagram for the meandered UC-EBG structure.

arrays, the use of a multi-layer substrate complicates the manufacturing process.

In this research, we propose a meandered, uniplanar compact EBG (UCEBG) structure. Utilizing meandered connecting lines, the equivalent inductance between EBG elements is significantly increased. Therefore, the proposed EBG geometry exhibits more compact dimensions and a wider bandgap frequency compared to the conventional U-EBG structure. A meanderline was employed to increase the inductance of the perturbed EBG structure [8]. However, the coupling reduction between array elements due to this meander-line EBG has yet to be reported. To investigate the performance of the proposed UC-EBG structure, a two-element antenna array with and without EBGs was fabricated and tested. The mutual coupling between the two patch antennas was measured to validate the suppression of the surface wave. The design, fabrication and measurement results are discussed in the following sections. 


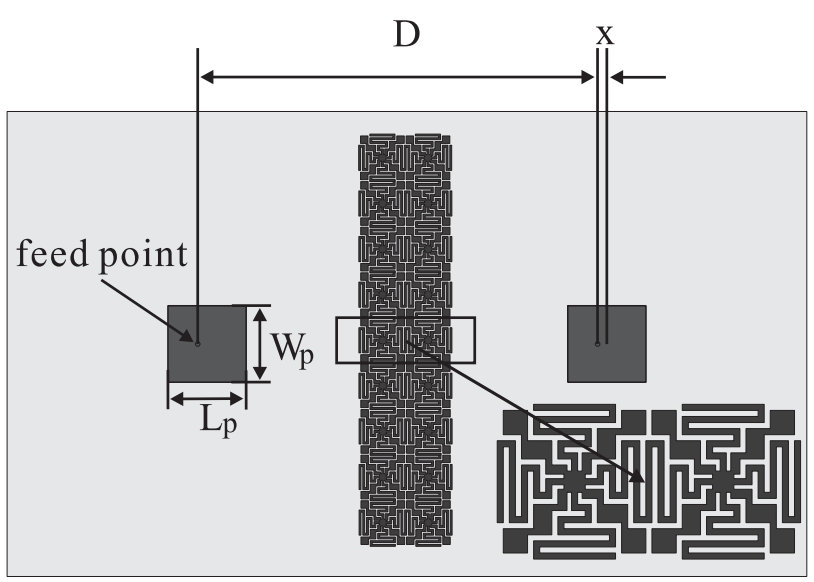

(a)

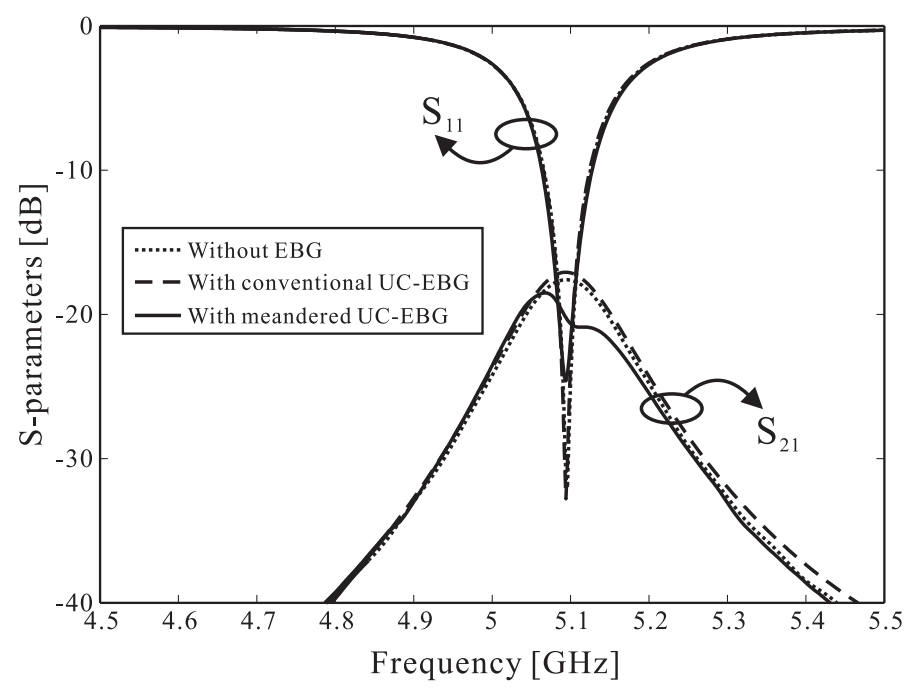

(b)

Fig. 2. (a) $1 \times 2$ microstrip patch antenna array with the proposed EBG structure (b) simulated reflection and coupling coefficients of the antenna array with and without EBG structure.

\section{EBG structure design}

The conventional U-EBG structure described in [4] and [6] is shown in Fig. 1(a). The unit cell includes a center metallic square patch attached to four small square patches at each corner. The adjacent cells are connected through thin microstrip lines. An EBG structure can be described as a lumped element circuit with distributed capacitors and inductors when the periodicity $(p)$ is small compared to the operating wavelength [9]. The inductance $L$ results from the current flowing through the thin microstrip line. A gap between the conductor edges of two adjacent cells introduces the equivalent capacitance $C$. Hence, the center frequency of the band-gap 


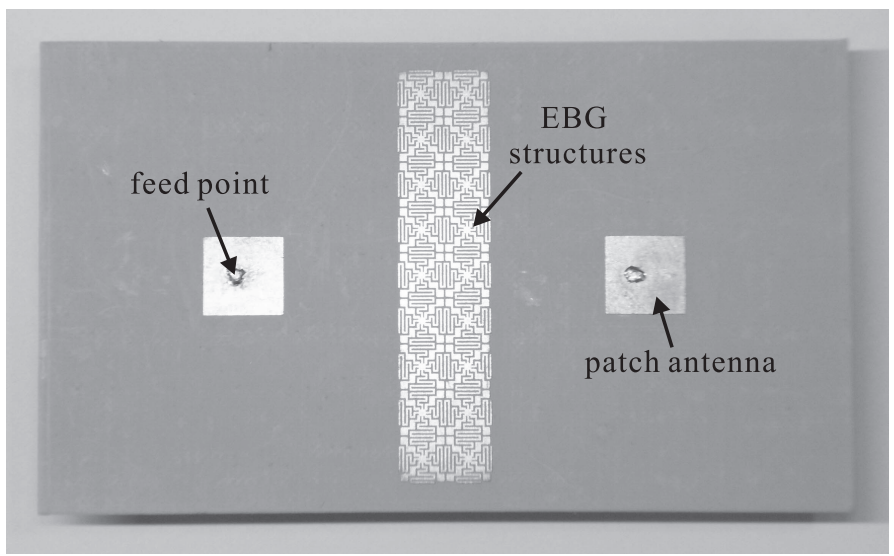

(a)

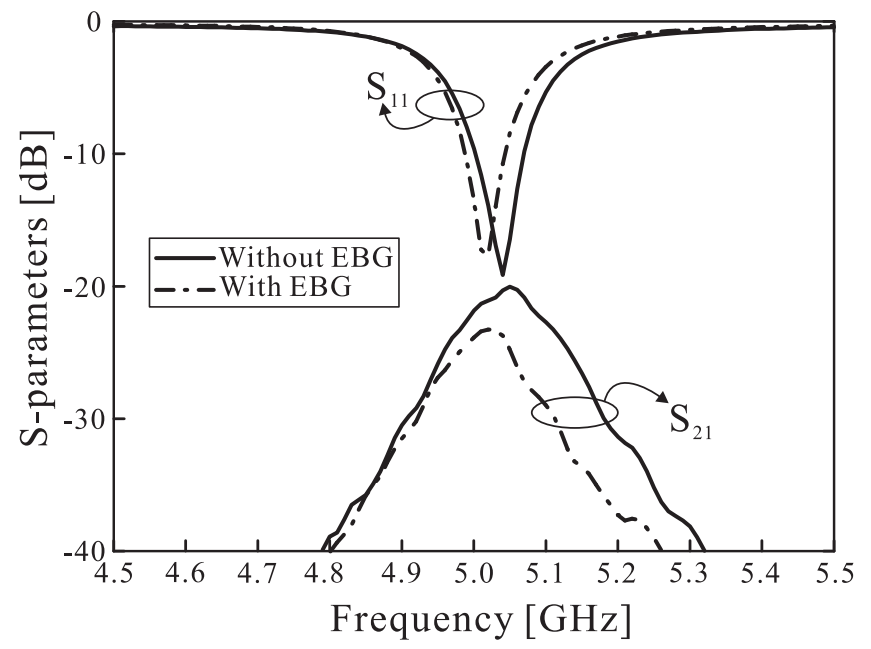

(b)

Fig. 3. (a) Photograph of the fabricated antenna array with the proposed EBG structure (b) measured reflection and coupling coefficients of the antenna array with and without the proposed EBG structure.

(resonant frequency of the $L C$ circuit) is calculated as follows [10]:

$$
f_{c}=\frac{1}{2 \pi \sqrt{L C}}
$$

The bandwidth of the band-gap frequency is given by

$$
B W=\frac{1}{\eta_{0}} \sqrt{\frac{L}{C}},
$$

where $\eta_{0}$ is the free space impedance. From (1), it is noted that the equivalent capacitance $C$ and inductance $L$ should be increased in order to decrease the center frequency of the band-gap. To do this, we introduce a UC-EBG structure with meandered connecting lines, as shown in Fig. 1(b). In the proposed EBG configuration, thin microstrip connecting lines between EBG cells were meandered to extend the current flow path, which increases the 
equivalent inductance $L$. Simultaneously, the equivalent capacitance $C$ decreases slightly due to the size reduction of the four square patches at the edge. This configuration not only reduces the center frequency, but it also widens the relative bandwidth of the band-gap according to (1) and (2). Therefore, it is expected that the compact and wideband EBG structure can be implemented using the proposed meander-line configuration. A proposed EBG structure and a conventional uniplanar EBG structure that operate at the same center frequency are designed to verify the attributes of the meandered connecting lines. The EBG structures were printed on a Taconic CER-10 substrate with a permittivity constant of $\epsilon_{r}=9.8$ and a thickness of $1.27 \mathrm{~mm}$. The parameters of the conventional U-EBG structure were chosen as follows: $p=4.9 \mathrm{~mm}, s_{1}=0.5 \mathrm{~mm}, d=2.1 \mathrm{~mm}, w=0.7 \mathrm{~mm}, g=0.2 \mathrm{~mm}$ and $w_{1}=0.2 \mathrm{~mm}$. For the meandered UC-EBG structure, the designed parameters are $p=4.9 \mathrm{~mm}, s_{1}=0.5 \mathrm{~mm}, s_{2}=1.5 \mathrm{~mm}, s_{3}=3.1 \mathrm{~mm}, d=2.0 \mathrm{~mm}, w=0.7 \mathrm{~mm}$, $g=0.2 \mathrm{~mm}$ and $w_{1}=w_{2}=0.2 \mathrm{~mm}$.

Figs. 1 (c) and 1 (d) show $k-\beta$ dispersion diagrams of the surface-mode propagation in the conventional EBG structure and in the proposed EBG structure, respectively. A simulation of $k-\beta$ dispersion was conducted using CST Microwave Studio (MWS) based on the three-dimensional finite integration time-domain (FITD) method. The first (dominant) surface wave mode is $\mathrm{TM}_{0}$, which has no cutoff frequency, and the second surface wave mode is $\mathrm{TE}_{1}$. One can observe a complete stop-band between the first mode, $\mathrm{TM}_{0}$, and second mode, $\mathrm{TE}_{1}$. The straight dotted lines represent propagation in a dielectric medium when $\epsilon_{r}=9.8$. The $\Gamma-\mathrm{X}$ branch represents $\beta_{x} p / \pi$ when $\beta_{y}=0$. The $\mathrm{X}-\mathrm{M}$ branch represents $\beta_{y} p / \pi$ when $\beta_{x}=\pi / p$. The $\mathrm{M}-\Gamma$ branch represents $\beta_{x} p / \pi$ when $\beta_{x}=\beta_{y}$. As shown in Figs. 1 (c) and 1 (d), the simulated band-gap frequency of the proposed meandered EBG structure is in the range of 3.9-5.94 GHz (41.5\%). This level of bandwidth performance is better than that of the conventional EBG structure $(5.02-6.82 \mathrm{GHz})$. Furthermore, the center frequency of the proposed design is lower than that of the conventional design, which indicates that miniaturization of the EBG structure was achieved without an increase in its dimensions.

\section{Application to patch antenna array}

E-plane coupled microstrip antenna arrays on a thick and high-permittivity substrates exhibit strong mutual coupling because the high permittivity and thick substrate easily launch surface waves which deteriorate the performance of such an antenna. Therefore, the proposed EBG structure was applied to suppress the surface waves, thereby enhancing the mutual coupling characteristic between the two patch elements in the E-plane. Fig. 2 (a) shows the two-element microstrip patch antenna array with the EBG cell array. The two-element patch array and a $2 \times 9 \mathrm{EBG}$ array are printed on the same upper layer of the dielectric substrate. The dimension of the patch was determined as $L_{p} \times W_{p}=8.45 \mathrm{~mm} \times 8.4 \mathrm{~mm}$ and it operates at a frequency of $5.1 \mathrm{GHz}$. The distance (D) from probe to probe is $43 \mathrm{~mm}$, corresponding to $0.73 \lambda_{0}$, 
where $\lambda_{0}$ is the wavelength in free space at the operating frequency. Each antenna patch is excited by a coaxial probe, which is located at a distance of $x=0.9 \mathrm{~mm}$ away from the patch center. All parameters of the proposed meandered UC-EBG structure were equal to those in Fig. 1 (b) so that the desired frequency band-gap can accommodate the resonant frequency of the antenna. The geometrical parameters of the conventional EBG structure shown in Fig. 1 (a) were designed to exhibit a center band-gap frequency identical to that of the meandered-EBG structure $(5.1 \mathrm{GHz})$. These parameters are $p=6.9 \mathrm{~mm}, s_{1}=2.7 \mathrm{~mm}, d=1.87 \mathrm{~mm}, w=3 \mathrm{~mm}, g=0.46 \mathrm{~mm}$ and $w_{1}=0.46 \mathrm{~mm}$. The simulated scattering parameters of the antenna array with and without the EBG structures are shown in Fig. 2 (b). The two patch antennas operate at the same resonant frequency near $5.1 \mathrm{GHz}$. A mutual coupling level $\left(S_{21}\right)$ of $-18 \mathrm{~dB}$ was observed in the antenna array without EBG structure. Note that there is no mutual coupling reduction after the insertion of the conventional EBG because the near-TEM mode is responsible for the coupling. Similar behavior was reported in earlier work [11]. On the other hand, the mutual coupling of the antenna with the meandered UC-EBG structure drops to $-22 \mathrm{~dB}$ due to the suppression of the surface wave. Therefore, not only is the miniaturization of the EBG structure achieved, but a mutual coupling reduction of $4 \mathrm{~dB}$ is also obtained by utilizing the proposed UC-EBG structure even if near-TEM mode coupling is dominant. A two-element array with the proposed EBG structure was also fabricated on a Taconic CER-10 substrate. Fig. 3 (a) shows an image of the fabricated antenna. The overall dimension of the antenna is $86 \mathrm{~mm} \times 50 \mathrm{~mm} \times 1.27 \mathrm{~mm}$. Fig. 3 (b) shows the measured scattering parameters of the antenna arrays with and without the EBG structure. It was found that the measured resonant frequency shifts slightly to a lower frequency compared to the simulation due to the fabrication tolerance. The measured results also show that the mutual coupling between the antennas fabricated with the EBG structure was reduced from $-20 \mathrm{~dB}$ to $-24 \mathrm{~dB}$ at the resonant frequency. This level of reduction in mutual coupling agrees with the simulated result shown in Fig. 2 (b).

\section{Conclusion}

In this paper, a UC-EBG configuration with a meander-line and its application to reduce the mutual coupling in arrays are presented. The proposed EBG structure was demonstrated to provide a more compact dimension and a wider frequency band-gap compared to the conventional uniplanar EBG structure. A dual-element patch array with and without an EBG structure was also fabricated and tested to validate the performance of the proposed EBG structure. The measured results demonstrate that mutual coupling reduction of $4 \mathrm{~dB}$ was achieved at the resonant frequency due to the effect of the EBG structure. Therefore, the proposed UC-EBG structure can be feasibly employed in compact antenna arrays to enhance the performance of these types of antennas. 


\section{Acknowledgments}

This research was supported by Basic Science Research Program through the National Research Foundation of Korea (NRF) funded by the Ministry of Education, Science and Technology (2012-0003052) 